\title{
INVESTIGATION OF FLOW CHARACTERISTICS OF A TURBULENT ANNULAR IMPINGING JET
}

\author{
M.S. ZAHRAN' AND R.M. KHATER ${ }^{2}$
}$$
\text { دراسة خوام الدلق المنطرب لنفاث حلقى يمـلدم بـطبع مسترى }
$$

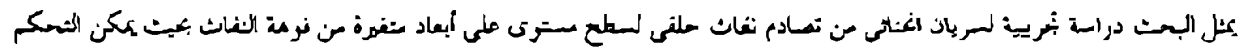

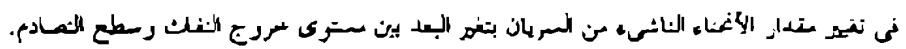

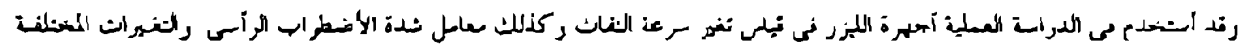

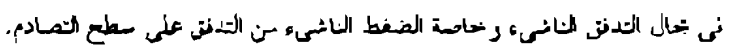

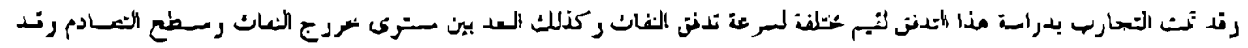

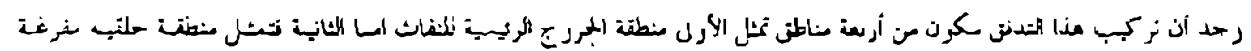

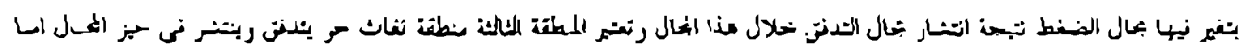

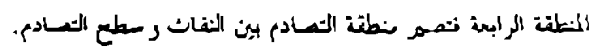

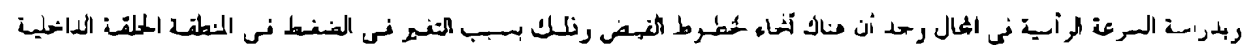

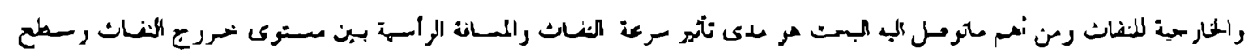 \\ إلنيسادم.
}

\section{ABSTRACT}

The structure of a curved flow created by an annular jet impinging on a nat plate has been investigated in the present experiniental model. As the curvature of the jet can be controlled by the gap between the jet exit and the impingement plate, hence the flow can be sinply analyzed in terns of the incernal pressure which is generated by the rate of change of nomentum due to the jet being turned through $90^{\circ}$. Measurements of the velocity profiles geross the jet and the pressure distribution along the impingement plate have been conducted for different jet curvalure and infet velocity. The velocity profiles across the jet and the turbulence parameters were measured using a Fiber Optics back scatlering Laser Dopples Anensometer (LDA) systein connected to a Burst Spectum Auralyzer (BSA) for data processing.

Examination of the velocity neasurements and pressure distribution along the impingenent plate indicated a strong curvature of the jet and high turbulence circulation and sivirl vithin various vortex regions across the now field. It is found that the difference in the jet entrained rate and the pressure

- ficld in the outer and the inner annular regions are responsible for the difference in tie displacencut of both the mean velocity and the turbulence intensity profiles outivard the central axis of the jet. The variation of the pressure field from sightely sub-atmospheric 10 a maxinum at the stagnation point and into the recirculation bubble is delected. The variacion of the normalized singnation pressure is carrelated wich the normalized ground heigin by a linear equation. within the range of the present experimental data.

\footnotetext{
'Mech. Engg. Dept., Shoubra Faculty of Engg., Togazig University ${ }^{2} \mathrm{Mech}$. Engg. Dept., Benha Higher Institute of Technology.
} 


\section{INTRODUCTION}

Impinging jets appear in various applications such as burning fluidic devices, and the flow field around Vertical or Short Takeoff and Landing (V/STOL) aircraft etc. An important consideration in the design of multiple jet for V/STOL aircraft is the aerodynamic interaction between airframe under surface and the ground in the presence of the lift jets. Also the behavior of turbulent flow fields produced by the interaction of multiple lif jets and a ground plane is strongly dependent on the lif jet nozzle geometry, nozzle exit spacing and orientation, jet exit conditions and nozzle exit height above ground.

Many literature are dealt with the modeling of this kind of flow but they are only concerned with the impingement of turbulent single jet emanating from circular or rectangular nozzles, notably the work of Donaldson and Snedecker [1]. The early experimental work of Miller [2] concerned the flow structure of dual free jets issuing into stationary air showed the sub-atmospheric pressure region due to entrainment in the central region of the jets. This region is also responsible for the convergence of the dual jets towards the center axis. Associated with the sub-atmospheric pressure region there is a recirculating vorex which recycled air in this region. But the annular jet is one of the most complex shear flow situations, in which two axisymmetric shear layers originating at the jet exit, one at the nozzle tip and the other at the center body. They eventually meet downstream and interact with each other.

The measurements of a basic free annular jet by Chigier [3] wcre a part of an investigation into the flow structure of coaxial concentric jets, their mean velocity and pressure measurements also established the formation of a sub-atmospheric region and a vortex in the center of the annular cavity of the jet. In addition the flow characteristics of the initial region of an annular jet discharging into stationary air have been investigated previously by [3-6]. Chigier and Beer [3]. Williams et al [4] studied them as a limiting case of a coaxial jet.

The configuration of the annular impinging jet adopted by the above workers does not contain any afterbody or bullet at the nozzle exit, an internal recirculating region was formed downstream of the interface [3-5]. This internal region was the result of the absence of any air supplies in the center and the entrainment of air from the main stream of the annular jet. In this recirculating region sub-atmospheric pressure and standing vortices were found. The extent of the standing vortices, the recirculating mass flow rate and sub-atmospheric pressure depended on the blockage ratio $\left(D_{i} / D_{0}\right)^{2}$.

The recent studies of annular jets concerned not only with the basic annular jet in which there is no bullet or afterbody in the center of the jet but also with the presence of such bullets [6-8]. Thus the flow issuing from the annular jet impinging on a flat plate can be simply analyzed in terms of increase in the internal pressure which is generated by the rate of change of momentum due to the jet turned through $90^{\circ}$.

The purpose of the present study is to investigate experimentally the effect of impingement of the annular jet on the flow field structure and turbulence characteristics in addition to the effect of the ground height on the pressure distribution over the impingement surface. 


\section{EXPERIMENTAL TECHNIQUE AND SETUP}

The experimental setup constructed for studying the configuration of the annular jet impinging on a circular flat plate is shown in Fig.(1). Air is drawn into the reservoir chamber by a three stage axial fan combination in which each stage is individually controlled to allow a wide range of test conditions. Inside the reservoir a number of honeycomb and wire screens were inserted to smooth the flow and reduce the turbuience level. Air is supplied directly to the annular nozzle which had a $30^{\circ}$ straight inlet profile to create a uniform velocity profile at the nozzle exit. The annular jet test section is formed as shown in Fig.(1). The exit jet nozzle diameter $D_{0}$ is 120 $\mathrm{mm}$, and the diameter of the center body, that was truncated at the jet exit plane, $D_{i}$ is $100 \mathrm{~mm}$. The impingement plate had a radius of $150 \mathrm{~mm}$ and its height above the jet exit was variable over the range $10-300 \mathrm{~mm}$. The impingement plate had a number of static pressure taps in the surface to measure the static pressure on the plate surface.

The velocity components and their fluctuating velocities across the jet at various vertical heights were obtained using a one component Back-scatter fiber Optic Laser Anemometer which was connected to a $25 \mathrm{~mW} \mathrm{He}-\mathrm{Ne}$ laser. The data acquisition arrangement is shown in Fig.(2); the He-Ne laser (1) passes light to the fiber optic head $\{2\}$ which is connected to a photo-detector $\{3\}$ which passes signals to the burst spectrum analyzer BSA $\{4\}$ and the oscilloscope $\{5\}$. The data output from the BSA is stored on the PC computer $\{6\}$ using BURSTWARE software; the PC is subsequently used to process and display the resultant velocity data. The PC is also used to control the automatic traversing mechanism through a control box $\{7\}$ and tracking system $\{8\}$ which carries the fiber optic head $\{9\}$. The two beams from the fiber optic head pass through a fixed focus lens giving a half angle of $4.6^{\circ}$ and the resultant measurement volume had dimensions of $1.87 \mathrm{~mm} \times 0.15 \mathrm{~mm}$.

The two beams were focused at the required position in the test rig $\{10\}$. An average of approximately 5000 values was obtained at each measurement location and a mean and standard deviation values were obtained at each point. The original and processed data was stored on the PC computer.

The air was seeded with atomized Ondina oil using an atomizer which produced particles of $5 \mu \mathrm{m}$ diameter. Seeding was introduced to the air in the reservoir, the central annular cavity and the external entrained air, in order to minimize seeding biasing.

\section{RESULTS AND DISCUSSION}

The domain of investigation is mostly confined as shown in Fig. (1). Measurements have been carried for an axial jet velocity of $38 \mathrm{~m} / \mathrm{s}$ and plate height of 0.05 and $0.1 \mathrm{~m}$. Furthermore experimental work has been carried for an axial jet velocity of $57 \mathrm{~m} / \mathrm{s}$ and plate height of $0.11 \mathrm{~m}$ to study the effect of jet velocity on the flow field turbulence structure and the pressure distribution along the impingement plate. 


\subsection{Jet Velocity Field structure}

The flow field structure of the circular single jet impinging to a flat surface, without considering the cross effect, can be divided into three zones [9-13]. The first is the free jet zone where the flow is the same as that of the jet issuing into an unbounded medium. The second is the impingement zone in which the large pressure field enhances the change of flow direction. The third is the wall jet zone, where the flow moves over the impinging surface due to the formed stagnation pressure. Besides the above description, it is known that the fluid surrounding the jet is entrained at the boundaries of the three zones.

In case of the basic annular jet [14-18], the flow field is divided into the initial merging zone, the intermediate and the fully merging zones. The nearest zone to the nozzle exit plane until roughly the plane, where the potential core disappears is called the initial merging zone. In this zone the internal recirculating vortex is found within the annular potential core. As in the case of single and coaxial jets the mean velocity within the annular potential core is the same as the jet velocity. Immediateiy downstream of the initial merging zone is the intermediate zone. It is within the region of the mixing flows from the annular potential core to the outer mixing region. Within the intermediate zone the axis of the annular potential core and the high mean velocity associated with the potential core merge at the central axis of the jet nozzle. The fully merged zone is the one downstream of the intermediate zone. In this zone complete merging of the flows from the initial merging zone has occurred. The fully merged flow behaves like a combined jet and its characteristics are similar to those of a single jet.

It is evident that in the case of the annular impinging jet the flow structure is quite different, either from the single impinging jet or the basic annular free jet discussed above. Thus, the annular impinging jet seams to be a combination of both. Studying the structure of the annular impinging jet obtained in the present work that it could be divided into four zones. The initial merging zone, the free jet $20 n e$, the impinging and the wall jet zones.

Though the above delineation of the four zones is approximate, it will be supported by similarity of the velocity and turbulence intensity profiles observed through these zones. .

Figure (3) presents the lateral distribution of the axial mean velocity ratio $\left(\mathrm{V} / \mathrm{V}_{0}\right)$ at different axial locations and Fig.(4) shows the contours of the axial mean velocity within the different zones of the jet flow field for different ground plate heights; namely 0.05 and $0.11 \mathrm{~m}$. From these figures it is found that the entrainment in the central zone (initial merging ) and the outer region of the annular jet is responsible for the generation of the recirculating vortex of toroidal form. This recirculating vortex is mainly found within the inner and the outer regions of the annular jet. Because of these vortices, the associated pressure difference from slightiy sub-atmospheric pressure in the inner region to the atmospheric pressure in the outer region. This radial pressure gradient draws the annular potential core outwards of the central axis causing a curvature to the jet streamlines. 
Figure (5) shows the radial distribution profiles of the axial turbulence intensity of the jet $\left(\sqrt{\left(v^{\prime}\right)^{2}} / v_{0}\right)$ at different axial locations of the flow domain.

Fundamentally, within the inner and the outer mixing regions, two turbulence intensity peaks are observed and as expected the lowest intensity is found across the annular core of the jet, these observations are detected for all axial locations. From the same figure the outer peak reduces while the other one increases, both with the increase of the axial position. In addition the peak locations in the outer mixing regions are displaced outward from the center line of the jet geometry due to the jet curvature, over the whole axial distance $1.15 \leq x / d_{c} \leq 1.5$. This observation has not been found in the free annular jet studied by [17] or the single impinging jet [9].

\subsection{Pressure Distribution on the impinging Plate}

At the same time during the velocity measurement data collection, the pressure distribution over the impinging plate is measured, Fig. (6-12), for different operating conditions. It is evident that the variation of pressure from slightly sub-atmospheric to a maximum at the stagnation point and into the recirculation bubble is well presented. Fig.(6) represents the effect of the ground height on the maximum pressure at the impingement point and also the position of this maximum value. But Fig.(7-12) show the effect of the initial exit velocity of the jet on the variation of the maximum pressure and its position with respect to the center line of the annular core of the jet. It is clear that the maximum pressure of the impinging surface is greatly affected by the ground height variation rather than the variation of the exit jet velocity. From these figures similarity of the pressure distributions at various jet velocities are well presented at different heights of the impingement surface.

Figure (13) shows the distribution of the stagnation pressure normalized by the inlet jet dynamic pressure $\left(1 / 2 \rho v_{0}^{2}\right)$ for six different impinging plate heights. It is noticed that the higher values of the stagnation pressure occurred at lower values of the ground height. This might be attributed to small radii of flow curvature. It is evident that the annular jet curvature tends to infinity for very large ground heights. As indicated from Fig.(I3), the variation of the normalized stagnation pressure with normalized ground heights can be correlated as :

$$
P^{*}=\left(0.131+2.92\left(\frac{h}{t}\right)\right)
$$

This correlation is presented in Fig. (13) compared with the measured data for three values of inlet velocity. The correlation gave a liner relation between the normalized stagnation pressure and the impinging height, which is in a good agreement with the measured data within the range of measurement. However, for very small values of $h / t$. tending to zero, the expected value of $P^{*}$ tends to infinity but in reality there will be no jet in this case. On the other hand for large values of $h / t$ the jet is changed into a free jet, which is not our case. 


\subsection{Entrained Air}

The entrained air was calculated from the measured data for the two cases of impinging heights $h=50 \mathrm{~mm}$ and $100 \mathrm{~mm}$. The obtsined results are plotted as normalized entrained air $\left(\dot{m}_{e}^{*}\right)$ versus normalized axial location $\bar{z}$. The entrained air is

normalized as: $\dot{\mathrm{m}}_{\mathrm{e}}^{*}=\dot{\mathrm{m}}_{\mathrm{e}} / \dot{\mathrm{m}}_{\mathrm{o}}(\overline{\mathrm{h}})^{\mathrm{n}}$

Where $\dot{m}_{0}$ is the inlet mass flow rate

$\bar{h}$ is the normalized impinging height $=h / d$, where $d$ the hydraulic annular diameter.

$\mathrm{n}$ an exponent is found equal to 1.2 from correlation.

While $z$ is normalized by the height $(h)$ itself.

The result depicted in Fig.(14) expresses the increase of the normalized entrained air with the increase of the axial location for different heights. This feature may be attributed to the increase of the annular jet curvature, and thus the increase of its cross section, with the increase of axial location as mentioned and shown in Fig. (3\&4).

The data is correlated and gave the following formula

$$
\dot{\mathrm{m}}^{*}=\mathrm{A}\left(1-\overline{\mathrm{e}}^{\overline{\mathrm{z}}}\right)^{2}
$$

where $A$ is a constant equals 3.0474

The comparison, shown in Fig.(14), between the obtained correlation and the measured data shows a good agreement.

\section{CONCLUSIONS}

In annular jet, the formation of the internal recirculating region and the inner mixing region has been detected. This is perhaps because of the surrounding effect of the annular potential core. From the mean velocity and turbulence intensity measurements the flow field can be divided into the initial merging (inner region of the annulus) free jet zone just downstream of the annular core of the jet, the impinging and the wall jet zones.

The difference in the jet entrainment rate and the pressure field in the inner and the outer recirculation regions are responsible for the difference in the displacement of both the mean velocity and the turbulence intensity outward the central axis of the jet geometry.

The ground height has a serious effect on the impinging pressure distribution on the impingement surface compared to the change of the exit velocity at the same impingement plane height. Simularity of the pressure distribution along the impinging plate at different exit velocities is found. An experimental correlation is developed in the range of the present data. 
The entrained air estimated from experimental data is correlated in terms of axial location in a generalized formula.

\section{NOMENCLATURE}

$D_{i} \quad$ Annular inuer diameter

D. Annular outer diameler

d Hydraulic annular dianierer

h Inipinging plate height above the cxit planc of the jet

$\vec{h}$ Normalized height $=1 \mathrm{l} d \mathrm{~d}$

|In|

[m]

[in]

(1)

$\dot{m}_{\mathrm{e}}^{*}$ Normalized entrained air mass now rate

(1)

mo Inlet mass flow rate

$n$ Exponent in equation $(1)=1.2$.

P. Stagnation pressure

P* Ps $0.5 \rho \mathrm{Vo}^{2}$ Static pressure

I Annular jet thickness

$\checkmark$ Jet velocity al lateral position

$V$, Axial velocity at the jet exit plane

$v^{\prime}$ Fluctuated axial velocity at lateral position

$x \quad$ Latcral distance

$z$ Axial distance from the jet exit plane

$\bar{z} \quad$ Normalized axial location $=2 \pi$. [kg/s]

\section{REFERENCES}

1. Donaldson.C du P. and, R.S., "A Study of Free Jer lmpingenem, Pant I - Mciul Propertics of" Frec and Impinging Jets," Journal of Fluid Mechanics, Vol. 45, Pt. 2 1971, pp. 281-314).

2. Miller D. and Coming E.W., "Force Monicnlum Fields In a Dual Jet Flow" J. Fluid Mech. Vol. 7. pp 237-256, (1)60).

3. Chigier N.A. and Beer J.M., "The Flow Region Near The Nozzle in Doubic Concenric Jels". Trans. ASME. J. Basic Eng. Vol. 86, pp 794- 804, 1964

4. William, T.J., Ali, M.R.M.H., and Anderson, J. S., "Noisc and Flow Cluacteristics of Coaxial Jets." Journal of Mechanical Engineering Sciencc, Vol. 11, 1969. pp. 133-142

5. Davies, T. W.. " A Study of The Aerodynamics of a Recirculation Zone Fonned in a Free Annular Air Jel," Ph.D. Thesis, University of Slseftscld, England, 1969.

6. Ko, N.W.M. and Chan. W.T.,"The Inner Region of Annular jeis" Journal of Fluid Mechanics, Vol, 93 1979, pp. 549-584.

7. Ko, N.W.M. and Lcung, K. C., "Covariance Mcasurements In The Iuicial Region of An Anmulisr Jct," Joumal of Sound and Vibration Vol. 80, 1982 pp. 339-354.

8. Ko, N.W.M. and Lam. K.M. "Further Measurements in The Inicial Region of All Almular Jet" Journal of Sound and Vibration Vol. 93, 1984 pp. 333-349.

9 C. J. Hwang and J.L. Lim, "Nunicrieal Study of Two Dinnensional lmpinging Jel Flow Ficld". AIAA J., Vol. 27 No 7, po 841-842, July 1989.

10 M. Wolfshtein, "Some Solutions of The Planc Iurbulent lmpinging Jet". Transictions of Tlse ASME. J. of Basic Engine, pp. 915-922, December 1970.

11 P.J. Russell and A.P. Hatto, "Turbulent Flow Characteristics of An Impinging Jet" Proc instr" Mecli. Engincers Vol 186,52, pp 635-644, 1972.

12 D.R. Kolansky and L. W. Glaze "Impingenicnt of Rectangulat Leis on A Ground Plane", AIAA J., Vol 20 No 5, pp 585-586. May 1982.

13 R.K. Aganwal and W. W. Bowver," Navier Stokes Computations of Turbulent Compressible Two Dimensional Inıpinging Jet Flow Fields", AIAA J., Vol. 20 No 5, pp. 577.584, May 1982. 
and Lain, K.M. "Flow Sirscturc of A Basic Amular Jel " AIAA J. Vol. 23 No 8 pp. 333-349. 1985

15. Ko. N.W.M and Lan, K.M. "Investigation of Flow Structure of A Basic Amular Jel" AlAA Vol. 24 No 9, pp. 1488-1493. 1986

16. Lam, K.M. Ko, N.W.M and Liu K. K." Wake and Wake Induced Shear Layer Excitation ill An Annular Jet " Phys. Fluids. Vol. 20 No 10, pp. $3121-3134.1986$

17. Ko. N.W.M. and Chan, W.T.," Similarity in The luitial Region of Amular Jels "Jourmal of Fluid Mechanics, Vol, 84 pan 4, pp 641-656. 1978

Tand Ko, N.W.M." Colyerent Struclure in The Oulcr Mixing Region of Annular Jes " loumal of Fuid Mechanics, Vol. 89 part 3. pp $515-533.1978$. 


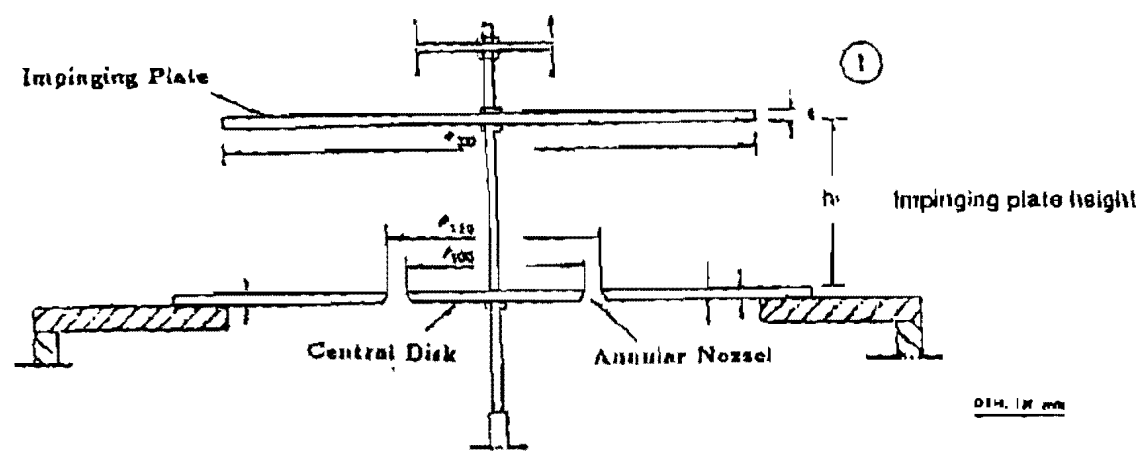

Teek section

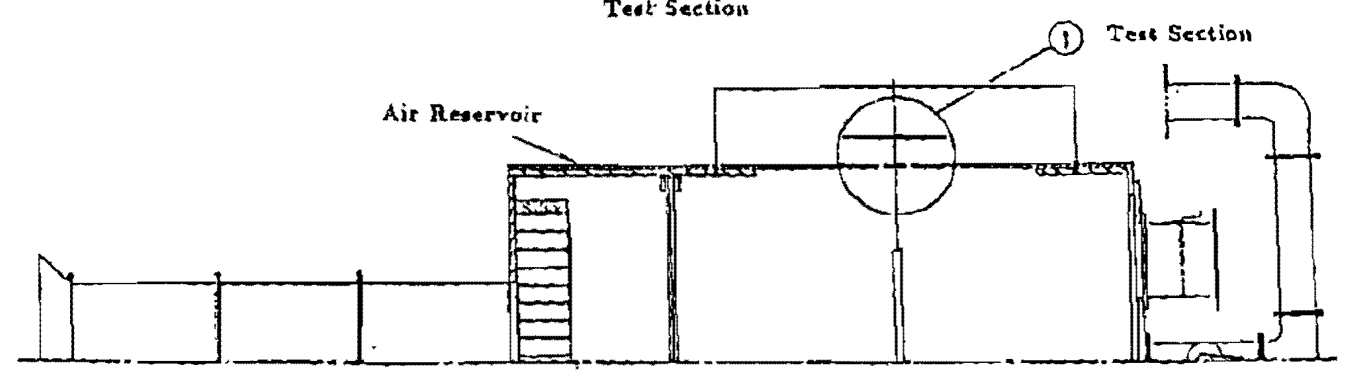

Fig.1: Schesuatic diagram of the experimentul seinp

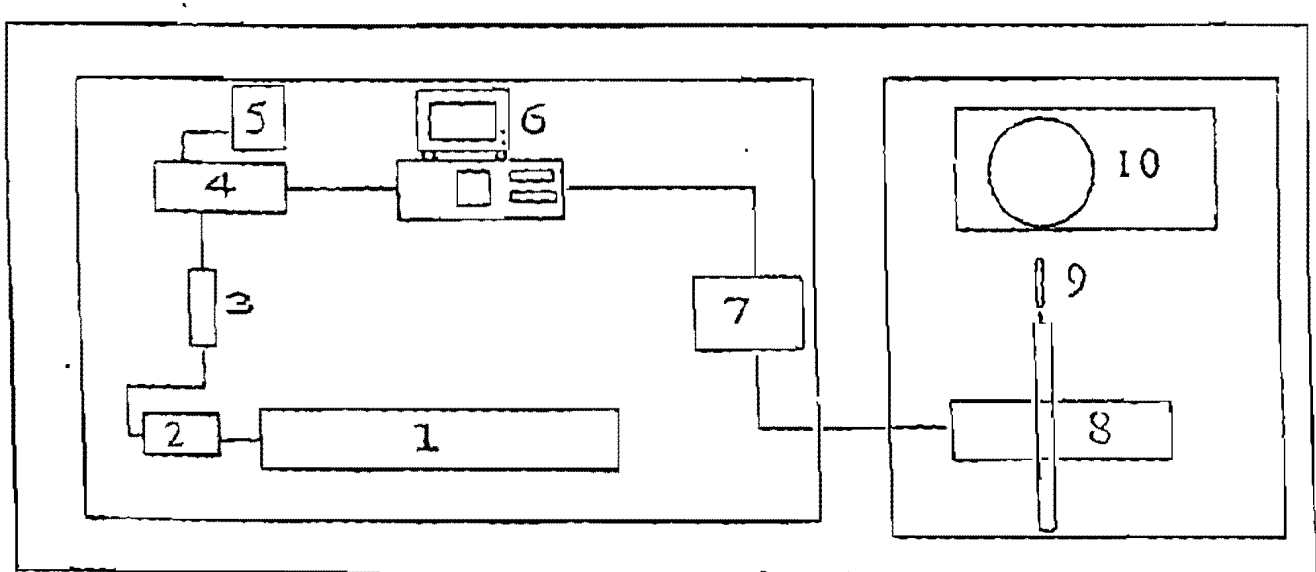

Fig.2: Schematic arrangement of the LDA 


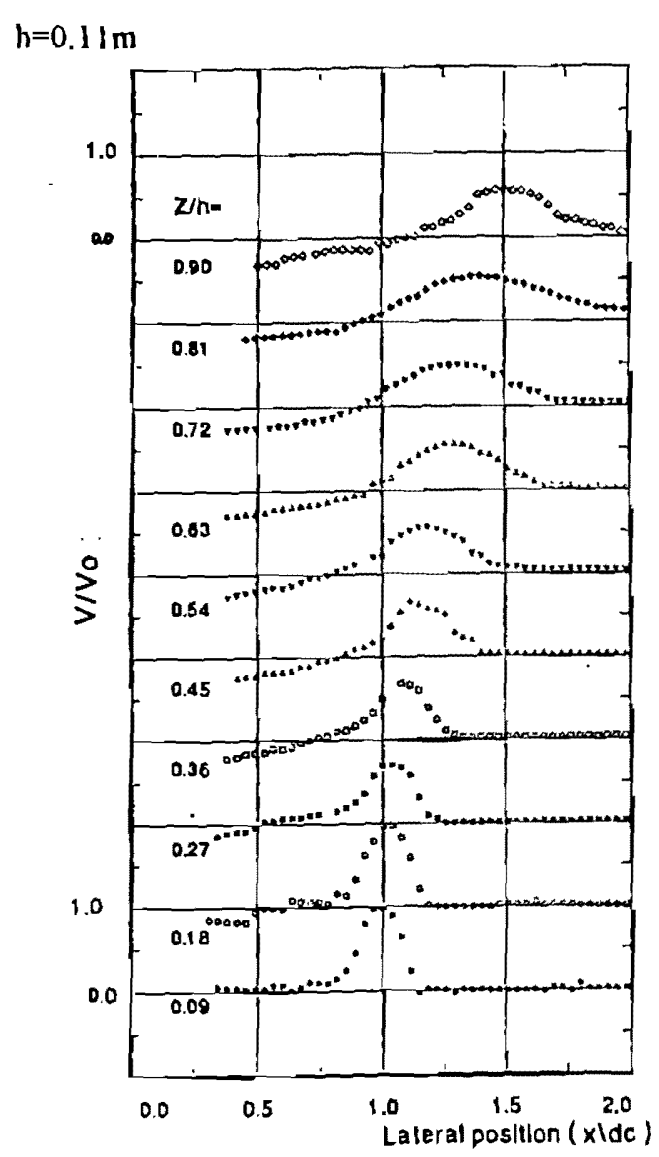

Fig. 3: Lateral distributions of the axin! jet velucity ratio $\mathrm{V} / \mathrm{Vo}_{0}$
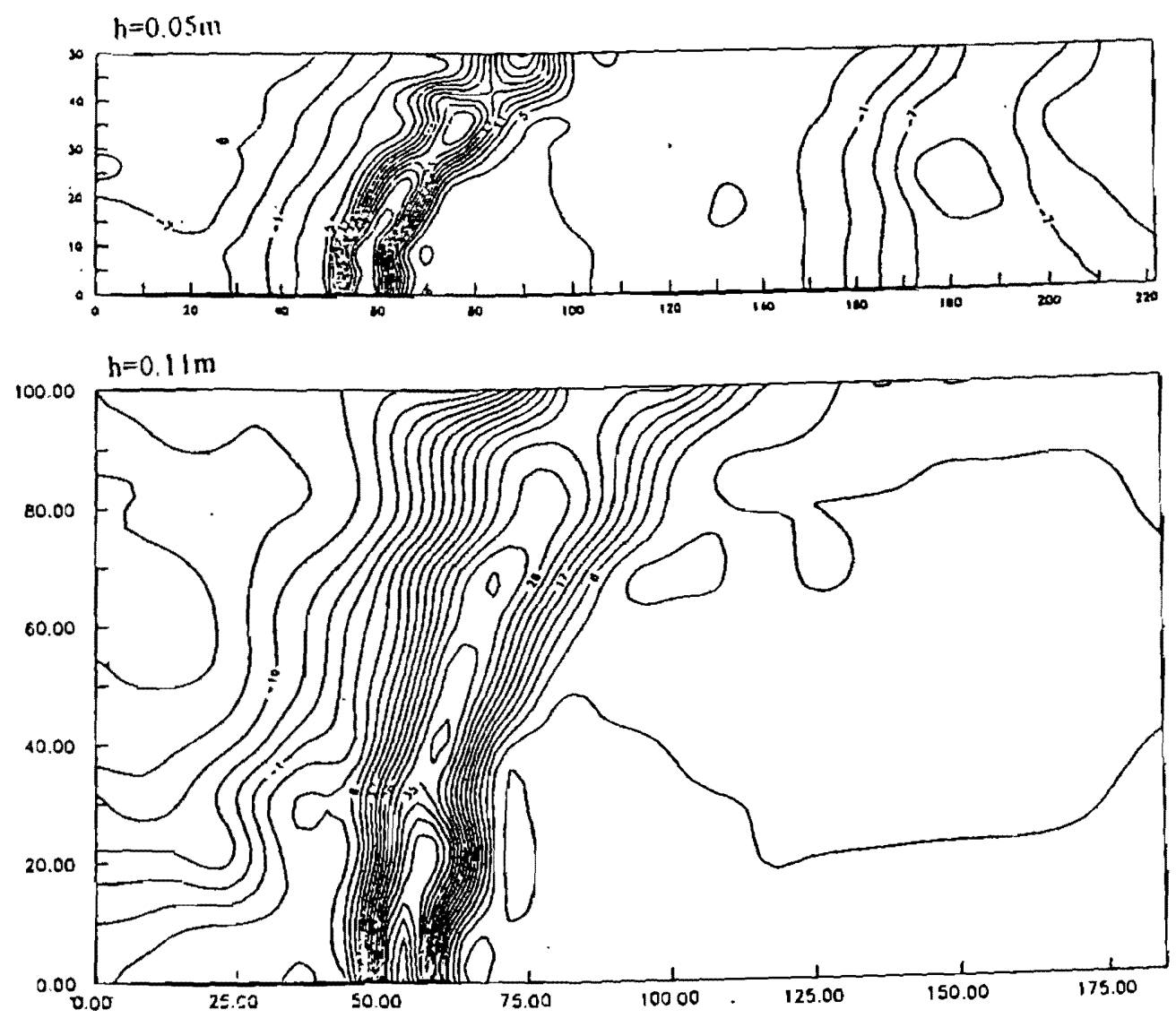

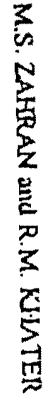

Fig.4: Axial velocity contours of the impinging annular jet 


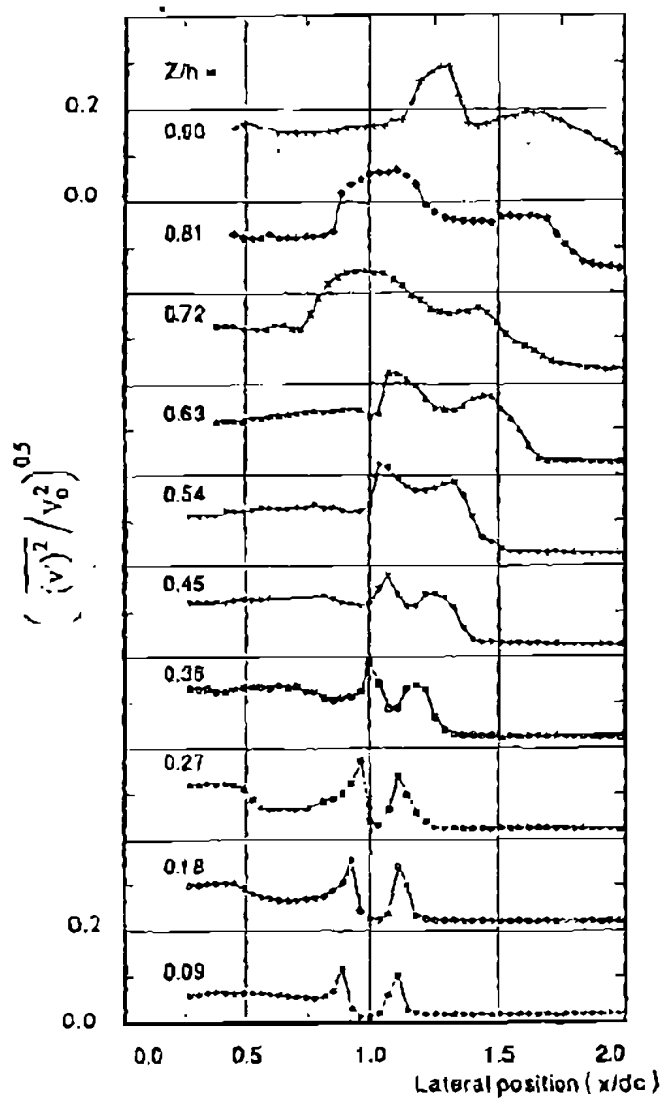

Fig.5: Lateral distributions of curbulence intensity of the axial velocity component

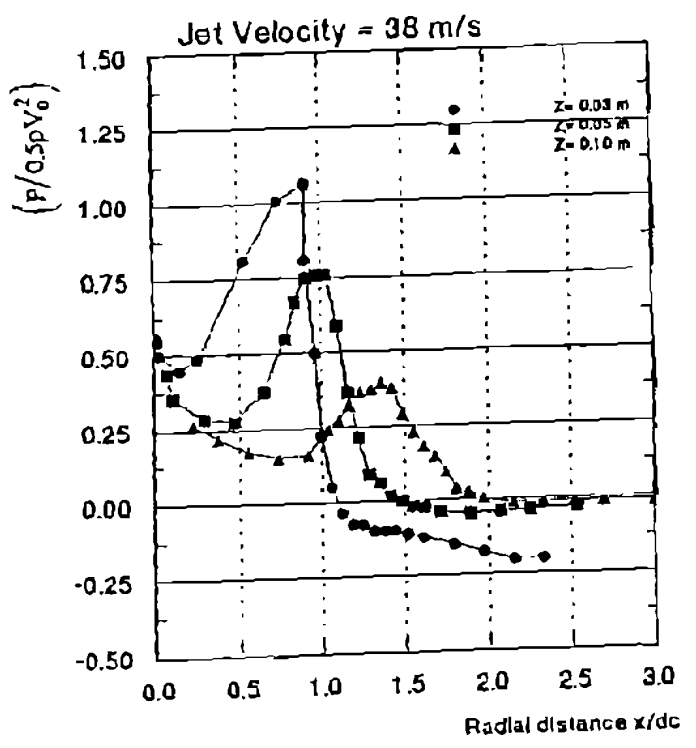

Fig.6: Pressure distributions over lle impinging flate 


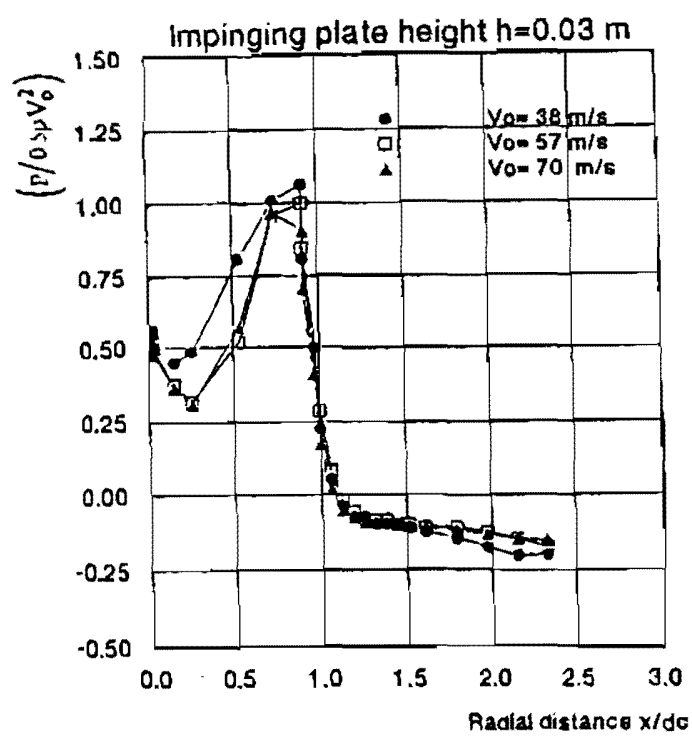

Fig.7: Pressure distributions over the impinging plate

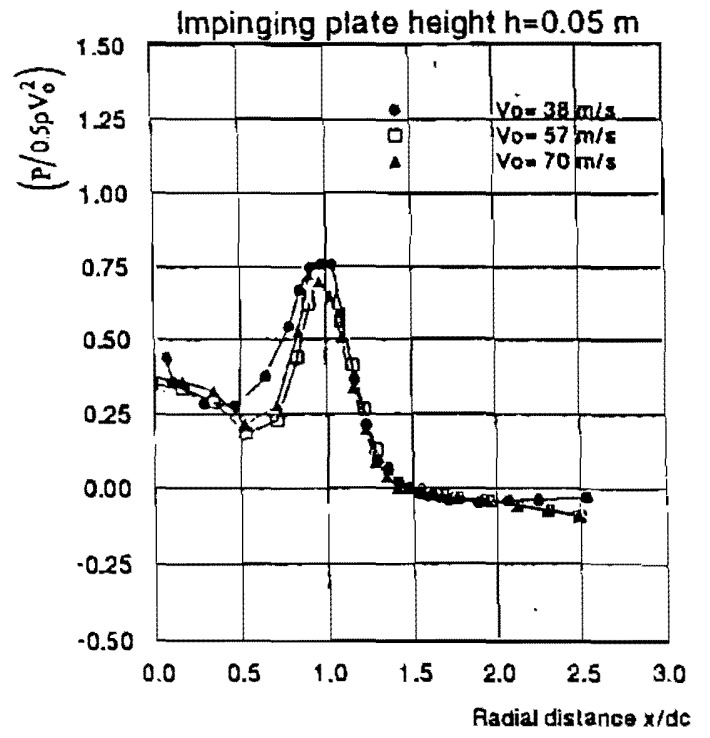

Fig.8: Pressure distributions over the impinging plate

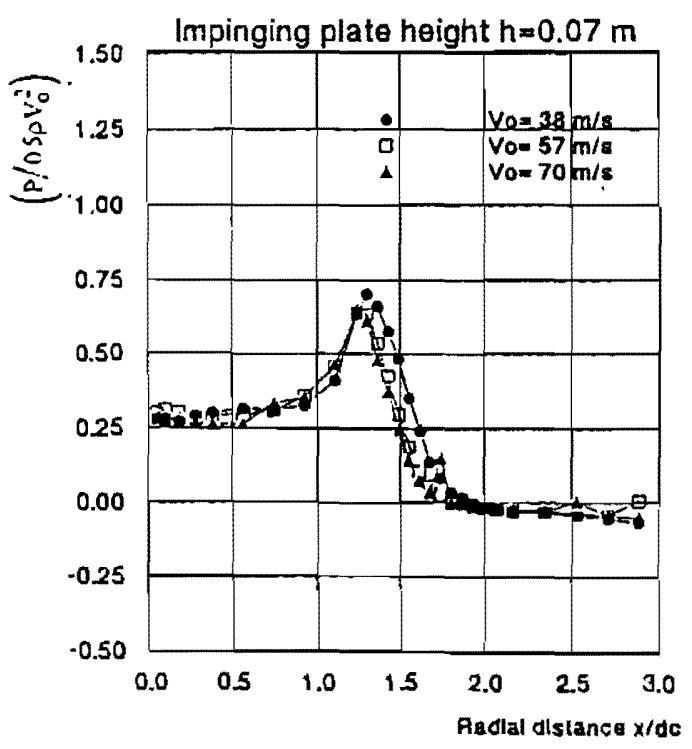

ig.9: Pressure distributions over the impinging plate

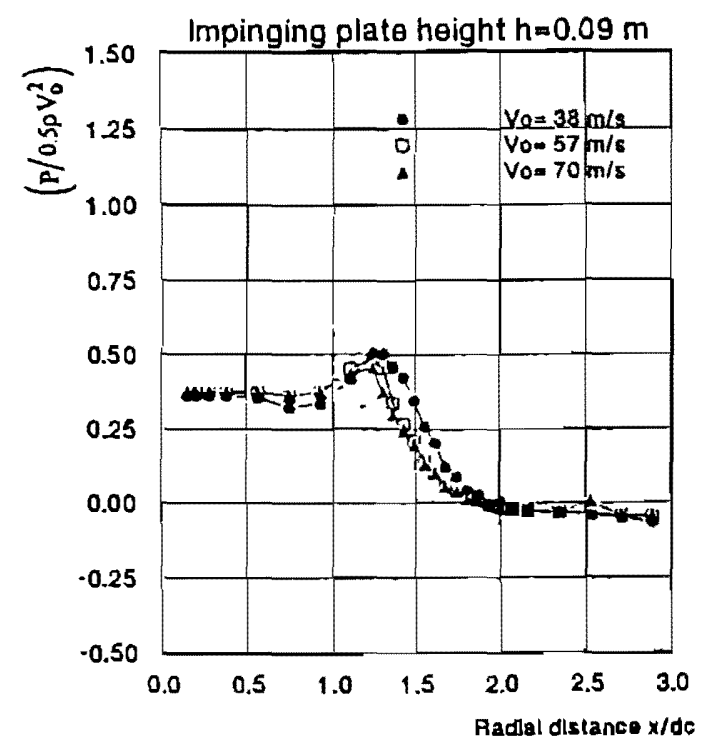

Fig. 10: Pressure distributions over the inpinging plate 


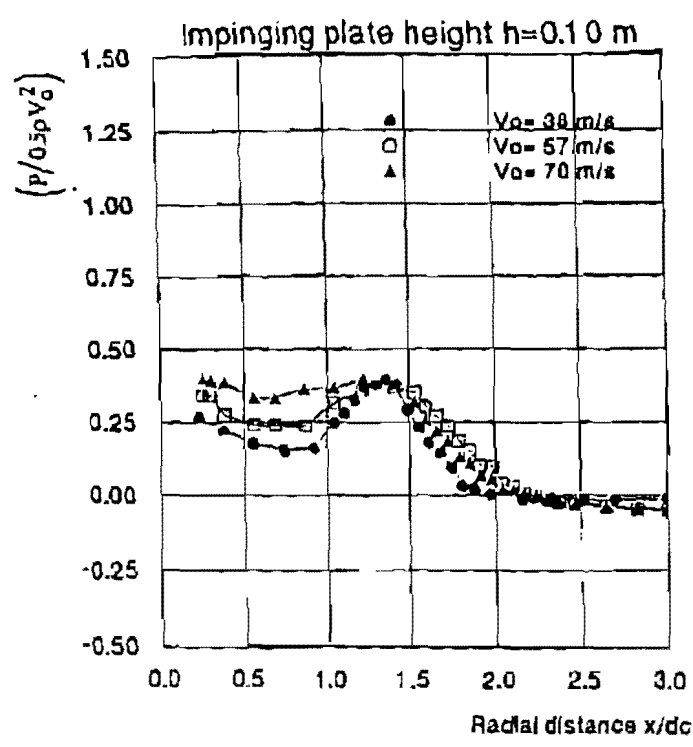

Fig.11: Pressure distributions over the impinging plate

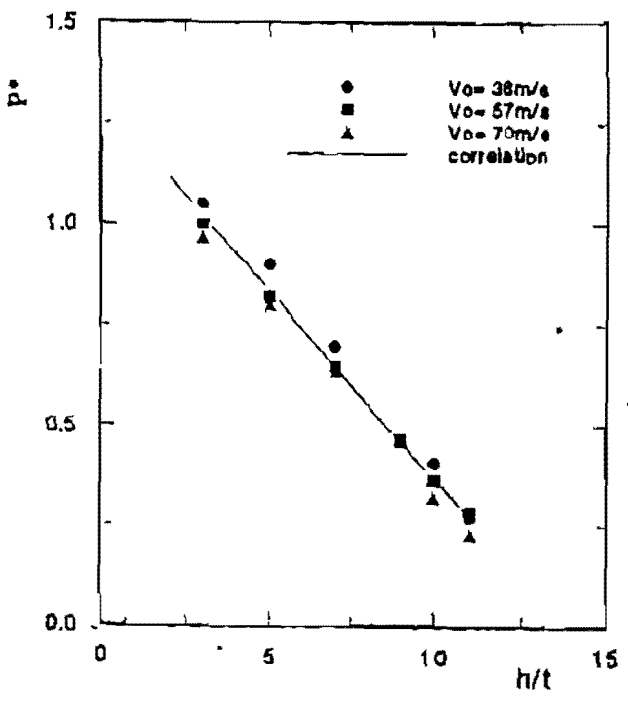

Fig. 13: Normalized stagnatiofi pressure correlation

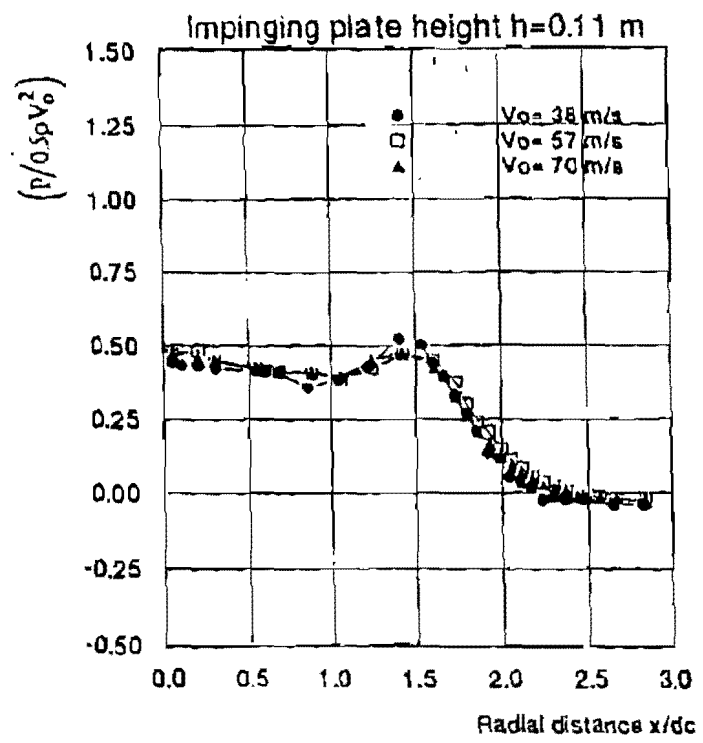

Fig. 12: Pressure distributions over lize impinging plate

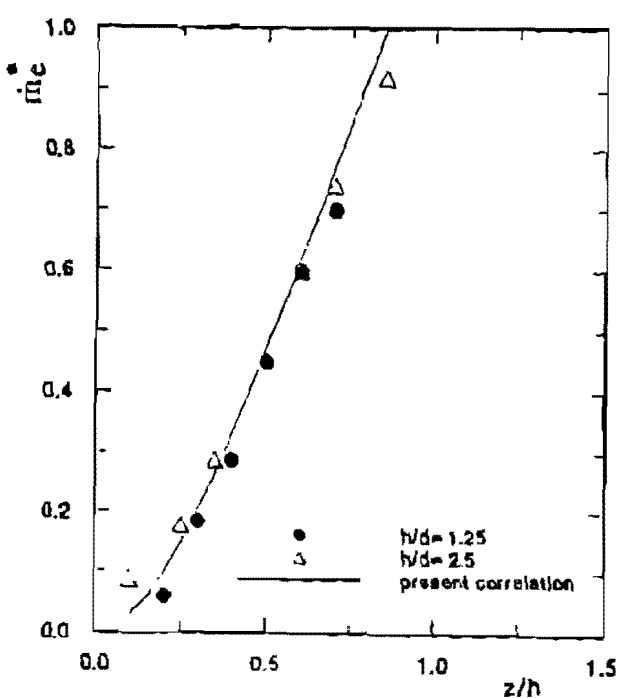

Fig.14: Comparison of experimental and curretuls entrained nir for differewt heights 